\title{
Operation of Modular Multilevel Converters Under Voltage Constraints
}

\author{
Mario López, Fernando Briz, Alberto Zapico, David Diaz-Reigosa and Juan Manuel Guerrero \\ Department of Electrical Engineering, University of Oviedo, Spain \\ lopezmario@uniovi.es
}

\begin{abstract}
MMCs are normally designed to operate in the linear region of the PWM. This limits the peak-to-peak phase voltage in the AC port to be lower than the DC port voltage. It is possible to increase the $\mathrm{AC}$ voltage beyond this limit by the use of overmodulation strategies. However, this is at the price of an increase in the harmonic content (THD) of the voltages and currents, and consequently, of a decrease of the power quality. While this type of operation is not desired in normal conditions, there are exceptional circumstances in which the MMC could be forced to operate in this mode. These would include transient anomalies, e.g. a temporary decrease of the DC port voltage or a temporary increase of the AC port voltage, or quasi-permanent conditions, e.g. the failure (and subsequent disconnection) of one or more cells in one or more arms of the MMC. Under this circumstances, the voltage margin between the $\mathrm{DC}$ and the $\mathrm{AC}$ port voltages required for the normal operation of the MMC might be lost. Consequently, the MMC should operate in the overmodulation region, or turned-off otherwise.

This paper addresses the use of overmodulation techniques in MMC under voltage constraints. Under these circumstances, the MMC control should guarantee stable operation, (i.e. a controlled power transfer between the $\mathrm{DC}$ and $\mathrm{AC}$ ports with the cell voltages maintained at their target values) and minimize the distortion of the currents, and consequently the adverse effect on the power quality.
\end{abstract}

Index Terms-Modular Multilevel Converter, MMC, overmodulation, power quality, zero sequence voltage

\section{INTRODUCTION}

Multilevel converters are a suitable option to cope with the new functionalities imposed by the power system operator, like power flow control, high power quality and reduction of transmission losses [1]. Among these, Modular Multilevel Converter (MMC) appears as a promising topology and it is a hot research topic nowadays.

MMC was first introduced one decade ago [2]-[4]. It realizes a bidirectional DC/AC power conversion, sharing the advantages of other multilevel converters: reduced size of filters due to better output voltage wave shape; lower switching losses due to the reduced switching frequency; capability of withstanding large terminal voltages using relatively low voltage power devices. Additionally, it provides attractive features compared to other multilevel topologies, such as modularity (identical cells

This work was supported in part by the Research, Technological Development and Innovation Programs of the Spanish Ministries of Science and Innovation and of Economy and Competitiveness, under grants MICINN10-CSD2009-00046 and MINECO-13-ENE2013-48727-C2-1-R, and by the European Commission FP7 Large Project NMP3-LA-2013-604057, under grant UE-14-SPEED-604057. are piled-up to increase the voltage), and consequently easy scalability; distributed energy storage, therefore eliminating the need of a bulk DC capacitor [2]-[5].

MMCs are normally designed to operate in their AC side in the linear region of the PWM. This limits the peak-to-peak voltage in the $\mathrm{AC}$ port to be lower than the DC port voltage. It is possible to increase the $\mathrm{AC}$ fundamental component of the voltage beyond this limit by using overmodulation strategies. However, this is at the price of an increase in the harmonic content (THD) of the voltages, and consequently in the currents and power. While this type of operation is not desired in normal operation, there are exceptional cases in which the MMC could be forced to operate in this mode. These would include transient conditions, e.g. temporary decrease of the DC port voltage below its rated value and temporary increase of the AC port voltage above its rated value, or quasi-permanent conditions, e.g. failure (and subsequent disconnection) of one or more cells in one or more arms of the MMC. Under these circumstances, the voltage margin between the DC and the $\mathrm{AC}$ port voltages required for the normal operation of the MMC might be lost. Consequently, the MMC will be forced to operate in the overmodulation region to maintain its power transfer capability, or be turned-off otherwise.

This paper addresses the use of zero sequence voltage components and overmodulation techniques in MMCs in the event of a mismatch between the DC and AC ports voltages, with the purpose of guaranteeing stable operation of the power converter, maintaining the power transfer capability between the $\mathrm{DC}$ and $\mathrm{AC}$ ports and minimizing the distortion of the currents and powers.

This paper is organized as follows. Basic concepts and power balance of the MMC are presented in Section II. Section III analyzes the voltage limits and overmodulation strategies for MMCs. Section IV discuss MMC operation under voltage constraints, while simulation results are presented in Section V. Finally, the conclusions are given in Section VI.

\section{MMC Model ANd Power Balance}

The analysis and methods proposed in this paper are specially well suited for three-phase MMCs (see Fig. 1). Furthermore, some of the overmodulation strategies that will be discussed are an extension of already existing methods for other types of three-phase power converters. It is useful therefore to use complex vector cells to represent the AC 


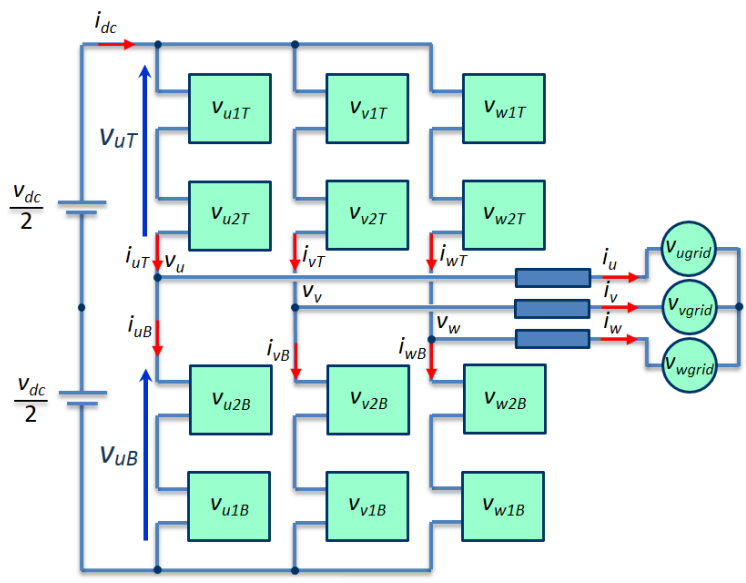

Fig. 1. Schematic representation of 3-phase grid connected MMC.

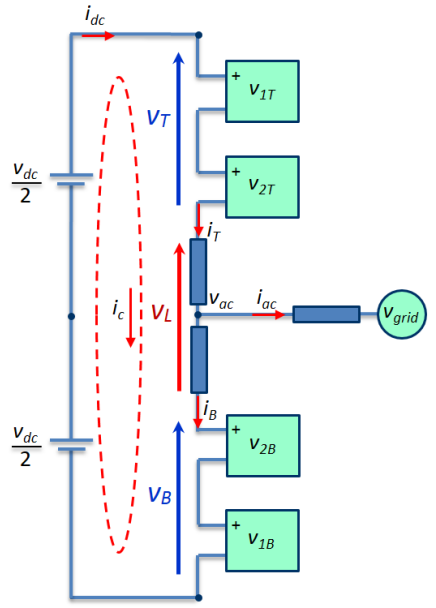

Fig. 2. Schematic representation of the MMC using complex vector notation.

variables of the MMC. These include AC port voltages and currents, as well as the $\mathrm{AC}$ component of the top and bottom arms voltages and currents.

The AC voltage and current vectors of the MMC are defined by (1) and (2) respectively, the same transformation can be applied to the AC component of the arms currents and voltages (3)-(6). By doing this, the three-phase MMC in Fig. 1 results in the equivalent MMC using complex-vector cells in Fig. 2.

$$
\begin{gathered}
v_{a c}=\frac{2}{3}\left(v_{u}+v_{v} e^{j 2 \pi / 3}+v_{w} e^{j 4 \pi / 3}\right) \\
i_{a c}=\frac{2}{3}\left(i_{u}+i_{v} e^{j 2 \pi / 3}+i_{w} e^{j 4 \pi / 3}\right) \\
v_{T}=\frac{v_{d c}}{2}-v_{a c}-\frac{v_{L}}{2} \\
v_{B}=\frac{v_{d c}}{2}+v_{a c}-\frac{v_{L}}{2} \\
i_{T}=i_{d c}+\frac{i_{a c}}{2}
\end{gathered}
$$

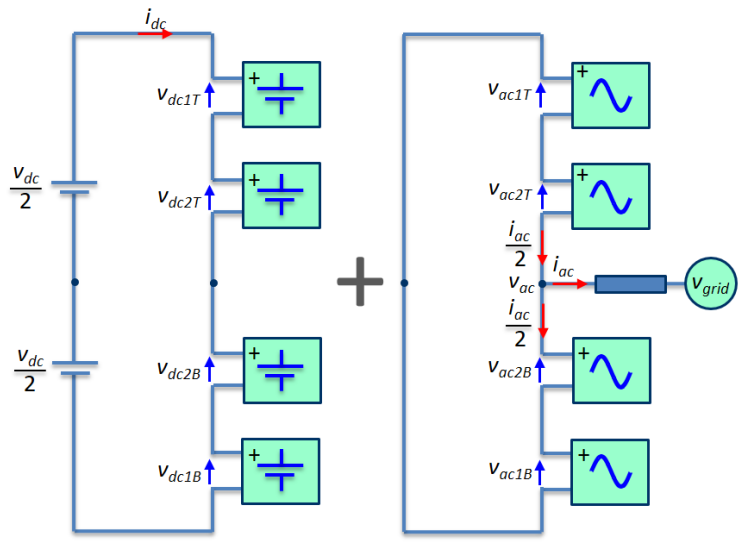

Fig. 3. DC (left) and AC (right) subcircuits of the MMC.

$$
i_{B}=i_{d c}-\frac{i_{a c}}{2}
$$

It is useful for analysis purposes to separate the MMC in Fig. 2 into its DC and AC subcircuits, as shown in Fig. 3. The voltage drop in the arm inductors has been neglected for simplicity. It is observed that the cells are series connected in the DC subcircuit (Fig. 3-left), the overall DC voltage of the cells corresponding to the DC voltage of the MMC (7).

$$
v_{d c n T}=v_{d c n B}=\frac{v_{d c}}{2 N} ; n=1: N
$$

On the contrary, for the AC voltage subcircuit (Fig. 3right), top and bottom arms are connected in parallel, the corresponding AC cell voltages being (8) and (9) respectively.

$$
\begin{aligned}
& v_{a c n T}=\frac{-v_{a c}}{N} \\
& v_{a c n B}=\frac{v_{a c}}{N}
\end{aligned}
$$

The AC current equally splits between the two arms. The resulting top and bottom arm currents are (5) and (6), while the circulating current is defined as (10). For the sake of simplicity, it is assumed that the circulating current does not contain harmonics [8][10], being therefore equal to the DC current.

$$
i_{c}=\frac{i_{T}+i_{B}}{2}=i_{d c}
$$

The limited energy storage capability of the MMC imposes that the power in the DC port has to be equal to the active power in the AC port (11) (losses neglected), with "**" standing for the complex conjugate. To achieve this, the DC current is controlled to adjust the power between DC and AC ports. The DC power in the cells (12) has to be therefore equal to the AC power (13) on average.

$$
\begin{gathered}
P_{d c}=v_{d c} \cdot i_{d c}=P_{a c}=\operatorname{Re}\left(v_{a c} \cdot i_{a c}^{*}\right) \\
P_{d c n T}=P_{d c n B}=\frac{v_{d c}}{2 N} i_{d c}
\end{gathered}
$$




$$
P_{a c n T}=P_{a c n B}=\operatorname{Re}\left(\frac{v_{a c T}}{N} \frac{i_{a c}{ }^{*}}{2}\right)=\operatorname{Re}\left(\frac{v_{a c B}}{N} \frac{-i_{a c}{ }^{*}}{2}\right)
$$

In addition, cell capacitor voltages must be kept at their target value. This is done by balancing methods, e.g. sorting algorithms [8][9] or individual balancing [7].

\section{Voltage Limits AND OVERMOdULATION STRATEGIES FOR MMCS}

\section{A. MMC Voltage limits}

A key figure in the design and operation of MMCs is the relationship between the $\mathrm{DC}$ and the $\mathrm{AC}$ voltages. Fig. 4 shows the MMC AC side voltage limits using complex vector notation in the $d-q$ plane.

A MMC with four cells per arm $(N=4)$ is considered. It is noted that this does not imply any lost of generality. Vertexes correspond to the voltage vectors that can be physically produced by the MMC, outer hexagon corresponding to the maximum voltage. As for other types of three-phase power converters, the maximum $\mathrm{AC}$ voltage that can be achieved will depend on whether zero sequence voltage components are used or not. The outer circle represents the voltage limit when a zero sequence voltage (triplen harmonics) [6] is added to the phase voltages. This corresponds to the physical voltage limit of the MMC in linear operation. The inner circle represents the voltage limit when triplen harmonics are not used. The use of triplen harmonics is seen to increase the voltage limit by an amount of $\approx 15 \%$. However, this is at the price of injecting a zero sequence voltage between the DC port and the neutral voltage of the $\mathrm{AC}$ port. The peak value of the phase voltage in the $\mathrm{AC}$ side of the MMC is equal to the magnitude of the AC voltage complex vector (1), being limited to $v_{d c} / \sqrt{3}$ when triplen harmonics are added.

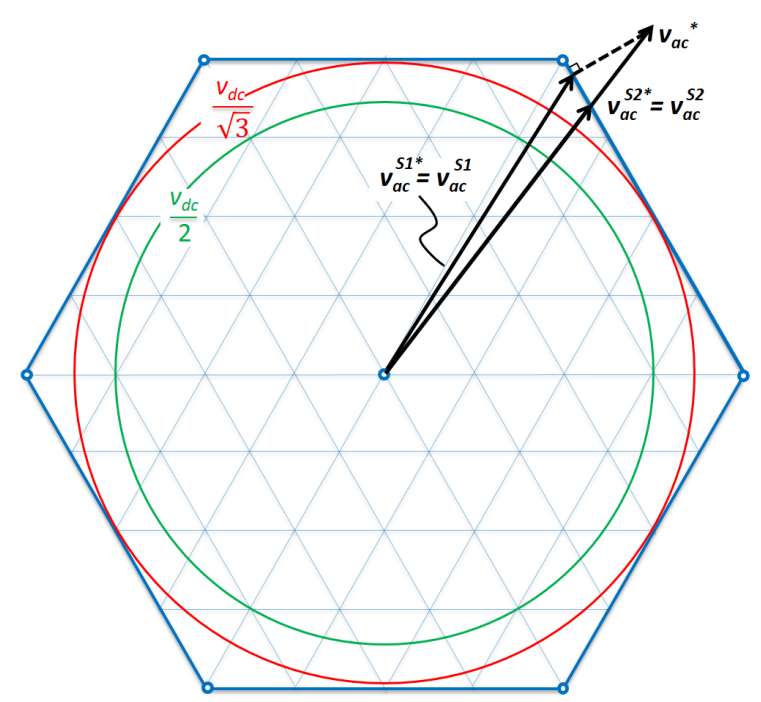

Fig. 4. Voltage limits and overmodulation strategies for a grid-connected MMC with 4 cells per arm. Outer circle: voltage limit in linear operation (requires the injection of zero sequence components to the individual phase voltage commands). Inner circle: voltage limit in linear operation without zero sequence voltage injection.
One difference between conventional three-phase converters and the MMC regarding the use of zero sequence voltage components, is that while in the first case the zero sequence voltage is obtained from a set of three phase voltages [6], for the MMC case six voltage references exist, i.e. top and bottom voltages (14)-(15) for phases $u, v$ and $w$.

$$
\begin{array}{ll}
v_{x T}^{*}=v_{d c T}-v_{a c x T}^{*}-\frac{v_{L x}^{*}}{2} & x=u, v, w \\
v_{x B}^{*}=v_{d c B}+v_{a c x B}^{*}-\frac{v_{L x}^{*}}{2} \quad x=u, v, w
\end{array}
$$

DC port voltage vs. AC port voltage is a key design aspect of the MMC. It is therefore useful for analysis and comparison purposes to define the ratio between the AC and DC voltages. This ratio indicates how much of the available DC bus voltage is actually used to produce the $\mathrm{AC}$ voltage. For a given $v_{d c}$ and $v_{a c}$, this ratio is (16) if triplen harmonics are added to the phase voltages, and increases to (17) (i.e. less margin between $v_{d c}$ and $\left.v_{a c}\right)$ if triplen harmonics are not used.

$$
\begin{gathered}
R_{t h i(p u)}=\frac{\sqrt{3} \cdot\left|v_{a c}\right|}{v_{d c}} \\
R_{n o t h i(p u)}=\frac{2 \cdot\left|v_{a c}\right|}{v_{d c}}
\end{gathered}
$$

Fig. 5 shows the reported ratio $R_{t h i}$ (in \%) vs. MMC power for already installed MMCs (or under development). It is interesting to note the dispersion of the data shown in this figure. Values of $R_{t h i}$ significantly lower than $100 \%$ means that the MMC has a large safety margin between its DC and AC ports voltages, and that therefore can likely operate within its voltage limits even in the event of anomalies, e.g. DC voltage lower than expected, or AC voltages larger than expected. Also this opens the opportunity to implement redundancy-based fault tolerant designs. I.e. it is possible to disconnect or by-pass faulty cells, without compromising the operation of the MMC. However, small values of $R$ also implies a misuse of the power devices, and of the cells in general. On the contrary, for values of $R$ closer to 100 , a better use of the cells and power devices is made, but at the price of an increased risk of forcing the MMC to operate without the required safety voltage margin. Finally, values of $R>100$ will

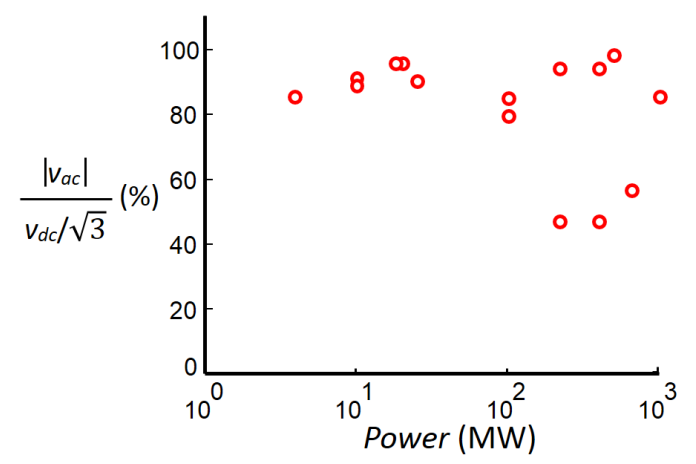

Fig. 5. AC-to-DC voltage ratio $R$ vs. power of already installed (or under development) MMC. 
impede the normal operation of the MMC. Depending on the severity of this anomaly and its duration, these effects can be manageable by the MMC control algorithms, or can produce an inadmissible degradation of the voltages (and currents) or even unstable operation, and consequently the disconnection of the MMC.

\section{B. Overmodulation strategies}

It is possible to increase the fundamental AC voltage supplied by the MMC with respect to the voltage limit in linear operation (outer circle in Fig. 4) by operating the MMC in the overmodulation region. This occurs whenever the commanded voltage vector is beyond the limit of $v_{d c} / \sqrt{3}$. Two different cases can be distinguished:

- If the AC voltage command $v_{a c}^{*}$ is outside the circle limit but within the hexagon limits, then the voltage command (on average) can be feasible.

- If the AC voltage command is outside the hexagon, then then the voltage command is not feasible.

An example of the second case is the voltage command $v_{a c}^{*}$ in Fig.4. Since this voltage command is permanently beyond the limits of the hexagon, it cannot be physically produced by the MMC. When this occurs, different strategies can be used to obtain a feasible voltage vector [6]:

- Minimum phase error: With this strategy, the magnitude is reduced to match the hexagon limits, the phase angle of the resulting voltage vector remaining unchanged. This corresponds to $v_{a c}^{S 2}$ in Fig. 4.

- Minimum error: The vector command is projected on the hexagon, the resulting feasible voltage vector being $v_{a c}^{S 1}$. It is noted that both magnitude and phase of the resulting voltage vector are modified with respect to the voltage vector command. This corresponds to $v_{a c}^{S 1}$ in Fig. 4.

Minimum phase error and Minimum error strategies allow to increase the fundamental component of the AC voltage compared to linear operation. However, this is at the price on an increase in the harmonic content of the AC voltage, and consequently of the currents and power. The physical limit for the fundamental component of the AC voltage would occur when the MMC operates in a six-step. The ratio $R_{t h i}$ can be increased with respect to the case of linear operation by an amount of $\approx 10 \%$ in this case. It is noted however that the six-step limit is unlikely to be used in practice, due both to power quality degradation as well as to MMC controllability issues.

\section{Overmodulation methods with sine-triangle modulators}

Space vector modulation representation has been used in the preceding discussion on overmodulation methods. However, sinte-triangle modulators are often used in MMCs. The dc-bus utilization by sine-triangle modulators can be increased to that of SVM through the use of zero sequence voltage components [6]. Centering the phase voltages with respect to the triangular carrier limits extends the maximum voltage in linear operation by $\approx 15 \%$. After injecting the homopolar component, the modulator will come into overmodulation when two phase

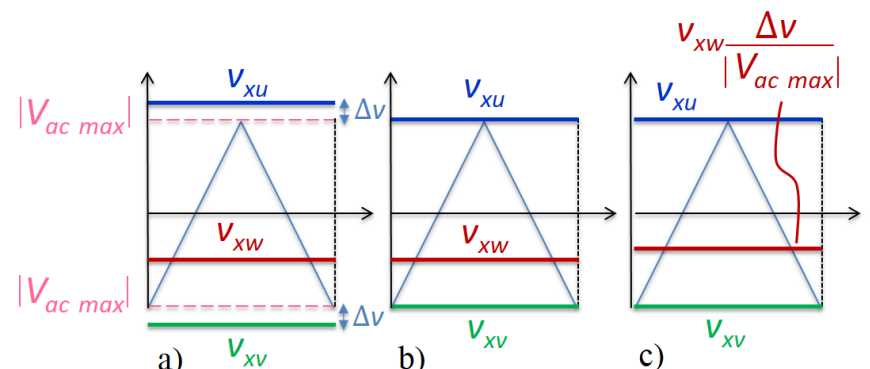

Fig. 6. a) zero sequence voltage injection. Unlimited voltage vector; b) Minimum error ( $v_{a c}^{S 1}$ in Fig. 4$)$; c) Minimum phase error $\left(v_{a c}^{S 2}\right.$ in Fig. 4).

voltages touch the triangular carrier limits. This corresponds to touching the hexagon limit in Fig. 4, it is schematically shown in Fig.6. If after injecting the homopolar component two phase voltages go outside the triangular carrier bounds $\left(v_{x u}\right.$ and $v_{x v}$ in Fig. 6-a), they will be physically limited ( $v_{x u}$ and $v_{x v}$ in Fig. 6-b and Fig. 6-c). If the third phase voltage remains unchanged ( $v_{x w}$ in Fig. 6-b), the resultant voltage vector will correspond to the minimum error SVM voltage vector. If it is reduced proportionally to the other two components reduction $\left(v_{x v}\right.$ in Fig.6-c), the minimum phase error SVM voltage vector is obtained.

\section{Operation of MMC Under Voltage CONSTRAints}

MMCs are designed to operate in the linear region. However, under exceptional circumstances, they can be forced to operate with voltage restrictions, i.e. without enough voltage in the DC link to provide the desired AC voltage. Three types of events that result in values of a voltage margin $R_{t h i}>1$ are considered:

- Decrease of the MMC DC voltage and/or increase of the MMC AC voltage (e.g. due to an increase of the grid voltage in grid connected MMCs) with respect to their rated values. Since the duration of such anomalies in the $\mathrm{DC}$ and/or $\mathrm{AC}$ voltages is in principle undefined, the MMC might need to withstand these operating conditions during relatively large periods of time.

- Sudden changes in the AC power, either due to changes in the power commands, or to disturbances in the AC side of the MMC. If this occurs, transients in the MMC voltage commands can produce a temporary lack of voltage. This situation should fade away after a relatively short time, but still will produce a transient disturbance in the operation of the MMC.

- Cell failure. One of the appealing properties of the MMC is its fault-tolerance capability. This can be achieved using redundant cells. If a cell fails in one arm, the remaining cells should be able to produce the commanded $\mathrm{AC}$ and $\mathrm{DC}$ port voltages and maintain the normal operation of the MMC. Ideally, the damaged cell would be replaced immediately and without discontinuing the operation. However, this might not be always the case in real practice. Failure of a cell in an MMC without spare cells, can force the MMC to operate with voltage 


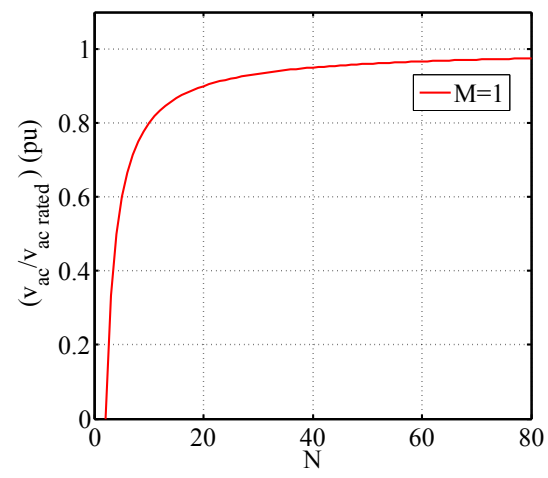

Fig. 7. Reduction of the AC port voltage limit vs. number of cells per arm $(N)$ in the event of one $(M=1)$ faulty cell.

constraints, or otherwise to discontinue its operation. In this case, the duration of the anomalies is in principle undefined, meaning that the MMC might need to withstand this operating conditions during a relatively large period of time.

The response of the MMC to these events will depend on the overmodulation strategy being used, as well as on the capability of the control loops to operate under voltage restrictions. Further discussion on this can be found in the next section.

An special case occurs when a cell failure forces the MMC to operate in overmodulation. The maximum AC port voltage that the MMC can supply as a function of the number of cells per $\operatorname{arm} N$ and the number of faulty cells $M$ is given by (18), the voltage decrease in the $\mathrm{AC}$ port with respect to the case of no faulty cells being (19).

$$
\begin{gathered}
v_{a c}=\left(\frac{1}{2}-\frac{M}{N}\right) v_{d c} \frac{2}{\sqrt{3}} \\
\frac{v_{a c}}{v_{a c \text { rated }}}=\left(1-\frac{2 M}{N}\right)
\end{gathered}
$$

Fig.7 shows the variation of the AC port voltage limit as a function of the number of cells per arm for $M=1$ (one faulty cell). AC port voltage will reduce proportionally to the number of faulty cells. It should be also remarked that when half the cells in one arm fail (i.e $\mathrm{M}=\mathrm{N} / 2$ ), there is no capability to produce $\mathrm{AC}$ voltage, since all the voltage is utilized in compensating the DC port voltage $v_{d c}$.

Two different strategies can be used when damaged cells limit the voltage capability of one phase:

- Operate the MMC asymmetrically, i.e. with different number of cells in the arms/legs.

- Maintain the symmetry among arms and legs, i.e. limit the AC voltage in the other two phases by disabling $M$ cells.

The resulting voltage limits are shown in Fig. 8. Fig. 8-left, shows the case when the MMC is operated with a different number of cells in each leg. Fig. 8-right shows the case when the same number of cells is disabled in all the phases. Comparing Fig. 8-left and Fig. 8-right, it is clear that the MMC can provide a significantly larger AC voltage in the first case,
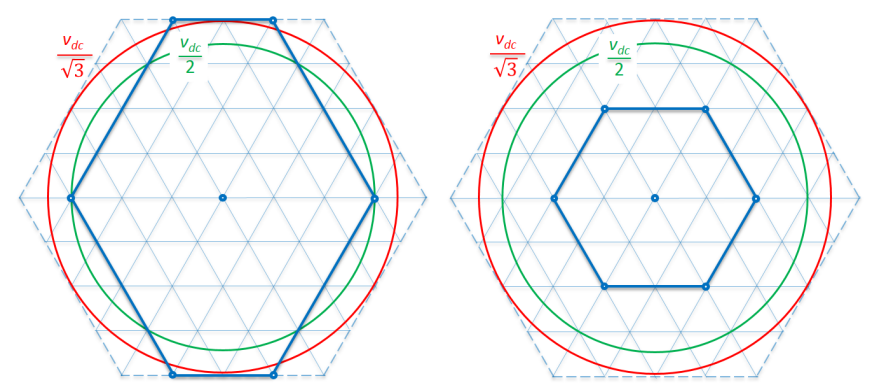

Fig. 8. Overmodulation limits in the case of a faulty cell. Left- Asymmetric legs: $N_{u}=3, N_{v}=N_{w}=4$ in the top and bottom arms of one leg respectively. Right- Symmetric arms and legs: $N_{u}=N_{v}=N_{w}=3$.

compared to the second case. However, this will produce an asymmetric behavior of the MMC, the harmonic content of the three phases being different. Simulation results showing this behavior are presented in the next section.

\section{Simulation Results}

Simulation results using the proposed concepts are presented in this section. The simulation setup consists of a grid connected MMC with four cells per arm $(N=4)$. Active and reactive powers for the $\mathrm{AC}$ side are commanded to the control. The same power profile will be used for all the simulations. Active power command increases linearly from 0 to $200 \mathrm{~kW}$ between $t=0$ and $t=1.1 \mathrm{~s}$. Homopolar harmonic injection is used.

Fig. 9 shows the AC active power, the fundamental component of $\mathrm{AC}$ voltage and the THD of the AC voltage vector, with the converter operating in normal conditions.

Fig. 10 and Fig. 11 show the MMC response when it is forced to operate into overmodulation, using Minimum error and Minimum phase error methods respectively. It is noted from the figure that the lack of voltage reduces the power transfer capability ( $P_{a c}$ is slightly smaller than $200 \mathrm{~kW}$ in steady state). However, operation of the MMC is perfectly
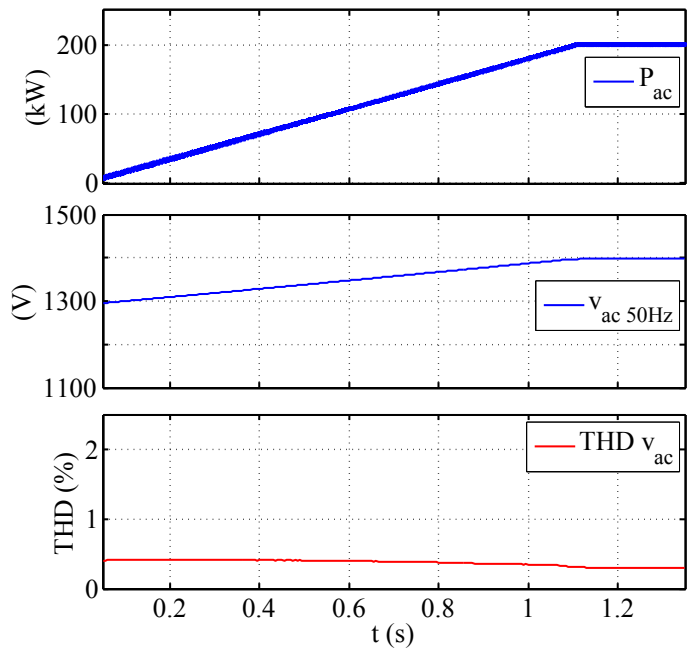

Fig. 9. MMC in normal operation, i.e. without going into overmodulation. From top to bottom: AC active power, fundamental component of AC voltage; THD of AC voltage. 

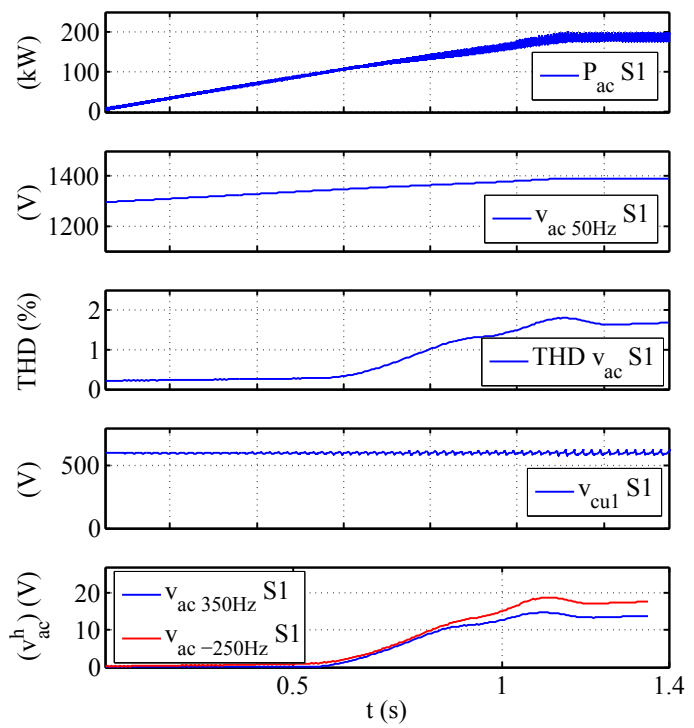

Fig. 10. Simulation results. Minimum error $(S 1)$ overmodulation method. From top to bottom: AC active power, fundamental component of AC voltage, THD of AC voltage, voltage of one cell capacitor, $350 \mathrm{~Hz}$ and $-250 \mathrm{~Hz}$ components of AC voltage.
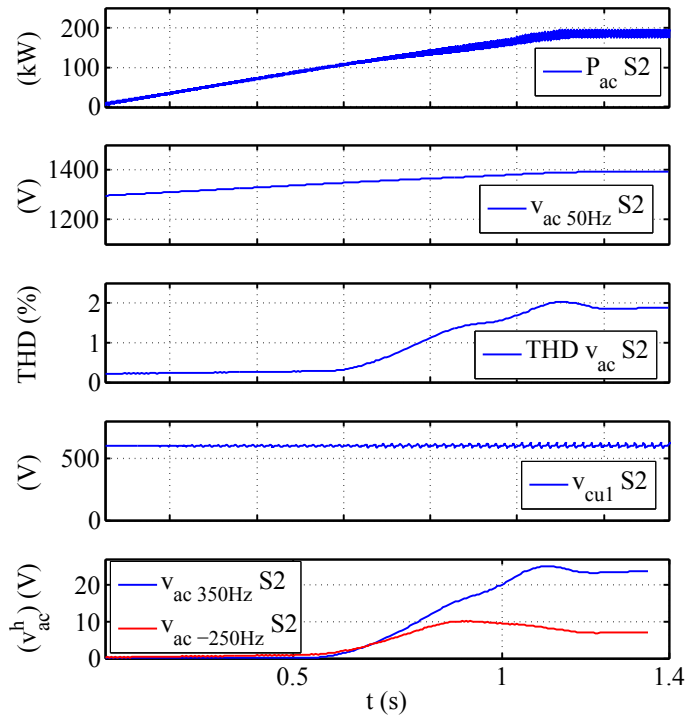

Fig. 11. Simulation results. Minimum phase error $(S 2)$ overmodulation method. From top to bottom: AC active power, fundamental component of $\mathrm{AC}$ voltage, THD of AC voltage, voltage of one cell capacitor, $350 \mathrm{~Hz}$ and $-250 \mathrm{~Hz}$ components of $\mathrm{AC}$ voltage.

stable. It is interesting to note that Minimum error method (Fig. 10) produces a lower distortion (smaller THD) and can transfer more power, compared to Minimum phase error (Fig. 11). It is also observed from the figures that the oscillation of the capacitor voltages slightly increases, but remains under control. The most relevant harmonic components of the AC voltage are at $-250 \mathrm{~Hz}$ and $350 \mathrm{~Hz}$, they are also shown in the figure.

It is concluded that both Minimum error both Minimum phase error saturation methods provide stable operation under voltage constraints. The oscillation of the cells capacitors voltage is maintained within reasonable limits, and without compromising the stability of the MMC.
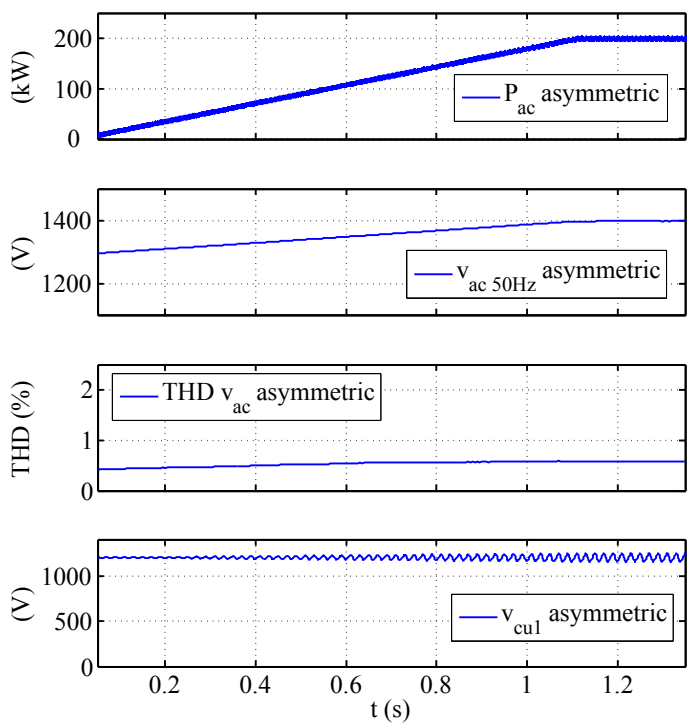

Fig. 12. Simulation results showing the effects of a faulty cell using Minimum error overmodulation, for the case of asymmetric operation of the MMC.
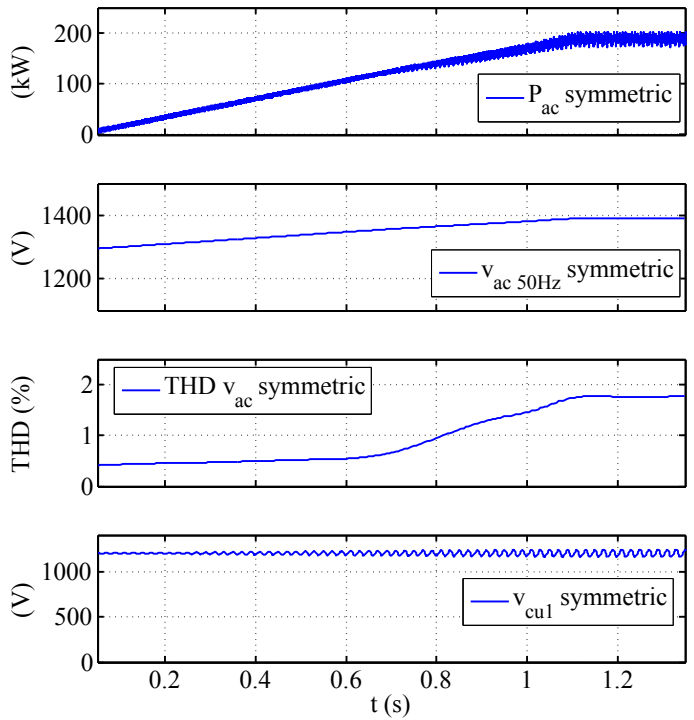

Fig. 13. Simulation results showing the effects of a faulty cell using Minimum error overmodulation, for the case of symmetric operation of the MMC.

Fig. 12 and Fig. 13 show the results when one cell in one arm fails. This forces the MMC to go into overmodulation. For the asymmetric case, the other two phases are not modified. This corresponds to the case shown in Fig. 8-left. For the symmetric case, one cell is removed from all the arms. This corresponds to the case shown in Fig. 8-right. As already mentioned, the asymmetric operation allows to obtain a higher output voltage (and consequently to transfer more power), and does not mean larger harmonics in the output voltage, since there is lack of voltage just in some sectors of the hexagon. It is important to note that by using the asymmetric case option, more power can be transferred and lower distortion is produced, compared to the symmetric case. Consequently, it is proved to be the best option. Similarly to the previous case, the oscillation of the cells capacitors voltage increase but within reasonable limits, and without compromising the 
stability of the MMC.

\section{CONCLUSIONS}

This paper analyzes the operation of the MMC under voltage constraints. This mode of operation can occur due to different reasons, including a decreased DC port voltage, an increased AC port voltage, or the failure of cells in one or more arms. To overcome this situation, it is possible to implement overmodulation techniques. By doing this, the MMC will be able to remain operative, but at the price of a decrease in the power quality.

Simulation results evaluating different overmodulation methods as well as strategies when failure of one cell occurs, have been provided. The combined use of Minimum error overmodulation with a realizable references strategy in the $\mathrm{AC}$ current regulator have been shown to guaranteeing stable operation of the MMC, providing good results.

\section{REFERENCES}

[1] J. Rodriguez, J.-S. Lai, and F. Z. Peng, "Multilevel inverters: A survey of topologies, controls, and applications," IEEE Trans. Ind. Electron., vol. 49, no. 4, pp. 724-738, Aug. 2002.

[2] A. Lesnicar, and R. Marquardt: An Innovative Modular Multilevel Converter Topology Suitable for a Wide Power Range, IEEE PowerTech Conference, Bologna, Italy, June 23-26, 2003

[3] M. Glinka and R. Marquardt: A New AC/AC Multilevel Converter Family, IEEE Transactions on Industrial Electronics, vol. 52, no. 3, June 2005

[4] A. Lesnicar, and R. Marquardt: A new modular voltage source inverter topology, EPE 2003, Toulouse, France, September 2-4, 2003

[5] Rohner, S.; Bernet, S.; Hiller, M.; Sommer, R.; , "Analysis and Simulation of a $6 \mathrm{kV}, 6 \mathrm{MVA}$ Modular Multilevel Converter," Industrial Electronics, 2009. IECON '09. 35th Annual Conference of IEEE , vol., no., pp.225-230, 3-5 Nov. 2009

[6] Briz, F.; Diez, A.; Degner, M.W.; Lorenz, R.D., "Current and flux regulation in field-weakening operation [of induction motors]," Industry Applications, IEEE Transactions on , vol.37, no.1, pp.42,50, Jan/Feb 2001

[7] Hagiwara, M.; Akagi, H.; , "Control and Experiment of PulsewidthModulated Modular Multilevel Converters," Power Electronics, IEEE Transactions on, vol.24, no.7, pp.1737-1746, July 2009

[8] Jae-Jung Jung; Hak-Jun Lee; Seung-Ki Sul, "Control strategy for improved dynamic performance of variable-speed drives with the Modular Multilevel Converter," Energy Conversion Congress and Exposition (ECCE), 2013 IEEE , vol., no., pp.1481,1488, 15-19 Sept. 2013

[9] Saeedifard, M.; Iravani, R., "Dynamic Performance of a Modular Multilevel Back-to-Back HVDC System," Power Delivery, IEEE Transactions on , vol.25, no.4, pp.2903,2912, Oct. 2010

[10] Hagiwara, M.; Hasegawa, I.; Akagi, H., "Start-Up and Low-Speed Operation of an Electric Motor Driven by a Modular Multilevel Cascade Inverter," Industry Applications, IEEE Transactions on , vol.49, no.4, pp.1556,1565, July-Aug. 2013 